\title{
WILEY-VCH
}

\section{Enhanced Dynamic Adhesion in Nematic Liquid Crystal Elastomers}

Takuya Ohzono ${ }^{1,2 *}$, Mohand O. Saed ${ }^{1}$, Eugene M. Terentjev ${ }^{1 *}$

Dr. T. Ohzono, Dr. M. O. Saed, Prof. E. M. Terentjev

${ }^{1}$ Cavendish Laboratory, University of Cambridge, J.J. Thomson Avenue, Cambridge, CB3 OHE, United Kingdom

${ }^{2}$ Research Institute for Electronics and Photonics, National Institute of Advanced Industrial Science and Technology (AIST) 1-1-1 Higashi, Tsukuba 305-8565, Japan

E-mail: emt1000@cam.ac.uk, ohzono-takuya@aist.go.jp

Keywords: liquid crystal elastomer, adhesion, viscous dissipation, dynamic soft elasticity,

Smart adhesives that undergo reversible detachment in response to external stimuli enable wide range of applications in household products, medical devices, or manufacturing. Here a new model system for the design of smart soft adhesives that dynamically respond to their environment is presented. By exploiting the effect of dynamic soft elasticity in nematic liquid crystal elastomers (LCE), the temperature-dependent control of adhesion to a solid glass surface is demonstrated. The adhesion strength of LCE is more than double in the nematic phase, in comparison to the isotropic phase, further increasing at the higher detachment rates. The static work of adhesion, related to the interfacial energy of adhesive contact, is shown to change very little within the explored temperature range. Accordingly, the observed enhanced adhesion in the nematic phase is primarily attributable to the increased internal energy dissipation during the detachment process. We correlate this adhesion effect with the inherent bulk dynamicmechanical response in the nematic LCE. The reported enhanced dynamic adhesion could lead to the development of a new class of stimuli-responsive adhesives. 


\section{WILEY-VCH}

In many applications of soft polymeric materials, the adhesive properties are important. In particular, pressure sensitive adhesives (PSA) ${ }^{[1,2]}$ form a class of applications of viscoelastic polymers, including elastomers and gels, which generally provide quick adhesion after applying light pressure, and detachment in some situations. However, in general, a polymer surface has fixed properties, being either sticky or non-sticky, and this varies only slightly and monotonically with the environmental conditions such as humidity or temperature. Thus, such general polymers are not suitable for 'smart' applications that require both adhesion and its release upon demand. Thus, the design of the system with dynamic adhesive characteristics, e.g. with small temperature change, is an important target in materials design.

Many systems have been proposed to have a (desirably) sharp transition of adhesive characteristics in response to various stimuli, including thermal, $\mathrm{pH}$, electric, chemicals etc. ${ }^{[3]}$ Many of them rely on the interfacial properties of the contact, such as surface topography and chemical functionality. The former directly affects the contact area $A$ to be detached and, therefore, the adhesion can indeed be altered by changing the surface topography. ${ }^{[4-7]}$ The latter mainly affects the interfacial energy density via the bare surface tension of each material or chemical/physical bond/entanglements. ${ }^{[8-10]}$ However, less attention have been paid to the use of the bulk viscoelastic characteristics, which are especially important in dynamic applications, towards stimuli-responsive adhesives. ${ }^{[11,12]}$ To our knowledge, no experimental study exists of the switchable adhesion of the nematic liquid crystal elastomers (LCEs), which possess unique bulk mechanical properties.

LCEs are a unique class of elastomers, in which the nematic director is coupled to the polymer network in both static and dynamic manner. ${ }^{[13]}$ In the macroscopically non-aligned LCE (called 'polydomain'), the local uniaxial nematic order $Q(T)$ develops in a usual manner of the frustrated first-order phase transition from the isotropic phase, ${ }^{[14]}$ but the nematic regions of a characteristic size of a micron are randomly mis-aligned in the bulk material. As a result, the polydomain LCE is macroscopically isotropic, ${ }^{[15]}$ but retains all its characteristic nematic 


\section{WILEY-VCH}

LCE parameters (such as the elastic modulus and loss factor, both relevant to this study). In contrast, the aligned ('monodomain') nematic LCE shows the uniform macroscopic anisotropy $Q(T)$ across the whole sample, and the characteristic effect of spontaneous shape change ${ }^{[16,17]}$ on increasing temperature $T$ across the nematic-isotropic transition temperature, $T_{\mathrm{NI}}$ (often called 'thermal actuation'). This effect makes LCE highly attractive for applications ${ }^{[13,18-22]}$ involving soft actuators, shape-memory materials, and others.

The other unique characteristics of nematic LCE, on which we especially focus here, is the effect of 'soft elasticity', which in dynamic-mechanical setting manifests itself in a large increase in the loss factor $\tan \delta$, and the decrease in the storage moduli $E^{\prime}$ and $G^{\prime} \cdot{ }^{[13,23-25]}$ This effect shows a great potential in LCE as energy damping materials. ${ }^{[23,26]}$ The higher internal viscosity originates from the additional nematic (orientational) interaction between polymer chains. This adds resistance against the local rotation of the liquid crystalline director relative to the network in response to the finite strain rate (similarly to the higher viscosity in the ordinary liquid crystals). The reduction in shear modulus of nematic LCE originates from the coupling of the antisymmetric part of shear strain to local nematic rotation, as in the Cosserat medium. ${ }^{[27,28]}$ Consequently, with a non-zero rate of deformation, the energy dissipation becomes much higher in the liquid crystal phase. Importantly, these viscoelastic properties are shown to have a close relationship with the performance of adhesives. ${ }^{[1,2,29,30]}$ As they can vary sharply across the nematic-isotropic transition in LCE, we expect them to alter the adhesion characteristics as well. This is a new concept (see Table 1 for comparison to other reported adhesive systems), with very little theoretical background so far: only one theoretical study of the effect of the nematic order in LCE on adhesion exists, in which the system was limited to a non-detachment case of PSAs. ${ }^{[31]}$ 
Table 1. Comparison of reconfigurable adhesion systems (non-solvent).

\begin{tabular}{|c|c|c|c|c|c|c|}
\hline Materials & $\begin{array}{l}\text { nematic LCE } \\
\text { (present } \\
\text { system) }\end{array}$ & $\begin{array}{l}\text { smectic } \\
\text { LCP }^{8,9}\end{array}$ & $\begin{array}{l}\text { elastomer } \\
\text { glass beads } \\
\text { composite }^{7}\end{array}$ & $\begin{array}{l}\text { elastomer } \\
\text { with hard } \\
\text { skin layer }^{5}\end{array}$ & $\begin{array}{l}\text { shape memory } \\
\text { polymer }^{4}\end{array}$ & $\begin{array}{l}\text { polymer } \\
\text { with azo } \\
\text { dye unit }{ }^{12}\end{array}$ \\
\hline Stimuli & thermal + light & thermal & strain & strain & thermal & light \\
\hline Transition type & $\begin{array}{l}\text { nematic- } \\
\text { isotropic }\end{array}$ & $\begin{array}{l}\text { smectic- } \\
\text { isotropic }\end{array}$ & $\begin{array}{l}\text { mechanical } \\
\text { deformation }\end{array}$ & $\begin{array}{l}\text { surface } \\
\text { buckling }\end{array}$ & $\begin{array}{l}\text { melting } / g \text { lass } \\
\text { transition }\end{array}$ & $\begin{array}{c}\text { molecular } \\
\text { isomerizati } \\
\text { on }\end{array}$ \\
\hline $\begin{array}{l}\text { Change affecting } \\
\text { adhesion }\end{array}$ & $\begin{array}{l}\text { bulk visco- } \\
\text { elasticity }\end{array}$ & $\begin{array}{c}\text { bulk } \\
\text { modulus and } \\
\text { surface } \\
\text { chemistry }\end{array}$ & $\begin{array}{l}\text { surface } \\
\text { topography }\end{array}$ & $\begin{array}{c}\text { surface } \\
\text { topography }\end{array}$ & $\begin{array}{l}\text { surface } \\
\text { topography }\end{array}$ & $\begin{array}{c}\text { bulk } \\
\text { modulus }\end{array}$ \\
\hline Adhesion test method & probe tack & probe tack & probe tack & $\begin{array}{l}\text { probe tack } \\
\text { (JKR) }\end{array}$ & probe tack & lap shear \\
\hline $\begin{array}{l}\text { Ratio of switchable } \\
\text { adhesions } \\
\left(F_{\text {hi }} / F_{\text {low }}\right)\end{array}$ & $\begin{array}{c}\sim 3 \\
\text { velocity } \\
\text { dependent }\end{array}$ & $\sim \infty$ & $\sim 4$ & $\sim 14$ & $\sim 3$ & $\sim 8$ \\
\hline $\begin{array}{l}\text { Maximum adhesion } \\
\text { strength }\end{array}$ & $\begin{array}{l}\sim 0.14 \mathrm{MPa} \\
\text { velocity } \\
\text { dependent }\end{array}$ & $\sim 0.6 \mathrm{MPa}$ & $\sim 4 \mathrm{kPa}$ & $\sim 60 \mathrm{kPa}$ & $\sim 30 \mathrm{kPa}$ & $\sim 1.7 \mathrm{MPa}$ \\
\hline
\end{tabular}

In this study, we experimentally demonstrate the ability to alter one of the tribological characteristics of nematic LCE, namely the adhesion between an elastomer and a flat glass probe, used as a representative example of solid. We evaluated the adhesion strength at different temperatures and speeds of detachment via probe-tack tests in the interfacial detachment regime. We find a strong temperature dependence in adhesion strength, measured by the maximum force before detachment $F_{\text {ad }}$. The results of adhesion testing are then correlated with the bulk dynamic-mechanical response of the LCE, and with the static interfacial energy of the adhesive contact investigated via contact-angle measurements. We found that the surface tension has very little temperature dependence, even across the nematic-isotropic transition. On the other hand, the dynamic soft elasticity makes the storage and loss moduli profoundly different between the two phases (it is, of course, also very different in the low-temperature glass phase, where no adhesion takes place). These results confirm that the observed change in the dynamic 


\section{WILEY-VCH}

adhesion is mainly induced by the phase-dependent bulk viscoelastic characteristics of the nematic LCE, suggesting a new strategy to design smart adhesives.

The adhesion of the polydomain nematic LCE was tested via the probe-tack experiment (Figure 1a and Experimental section for details). Our material was designed to have relatively low $T_{\mathrm{NI}}$, which makes adhesion experiments more practical within the temperature range of 20 $60^{\circ} \mathrm{C}$, and better suitable for applications, including household and biomedical products (see Experimental, and Figure $\mathbf{S 1}$ in the Supporting Information). ${ }^{[32-34]}$ Figure $1 \mathrm{~b}$ shows a typical force curve obtained by the probe-tack test using the thin LCE film, with the thickness of $d=0.1 \mathrm{~mm}$, and the probe area of $A=25 \mathrm{~mm}^{2}$. In our experiments, the sharp drops of the monitored force were always observed, and the repeated probe-tack curves were highly reproducible, indicating the interfacial detachment without fatal fracture of the bulk LCE under the present test conditions.

The maximum force required to detach the probe from the sample is defined as the 'adhesion strength' $F_{\text {ad }}$, and its dependency on the probe speed $v$ relative to the sample is shown in Figure 1b, comparing the room temperature (RT, $\left.20^{\circ} \mathrm{C}\right)$ and a high temperature (HT, $\left.60^{\circ} \mathrm{C}\right)$. The force ratio $R_{\mathrm{Nem} / \mathrm{Iso}}=\frac{F_{\mathrm{ad}}(\mathrm{RT}, v)}{F_{\mathrm{ad}}(\mathrm{HT}, v)} \sim 3$. The increase of $F_{\mathrm{ad}}$ with increasing $v$ points at the important role of the bulk viscoelasticity in the adhesion process. The temperature dependence of $F_{\text {ad }}$, shown in Figure 1c, indicates that the adhesion strength is closely related to the phase behavior of the LCE. The LCE is nematic at RT, and isotropic at HT, which is confimed by the dynamic scanning callorimetry (DSC) (Figure 2a) and wide angle X-ray scattering (WAXS) (Figure S2 in the Supporting Information). The probe-tack test results show a tangible difference in the adhesion between the two different phases. Although the physical relationship between the nematic order parameter and the adhesion is not yet established, it is noteworthy that the curve roughly follows the critical behavior of the phase transition of the nematic LCEs. ${ }^{[13,25]}$ 
WILEY-VCH
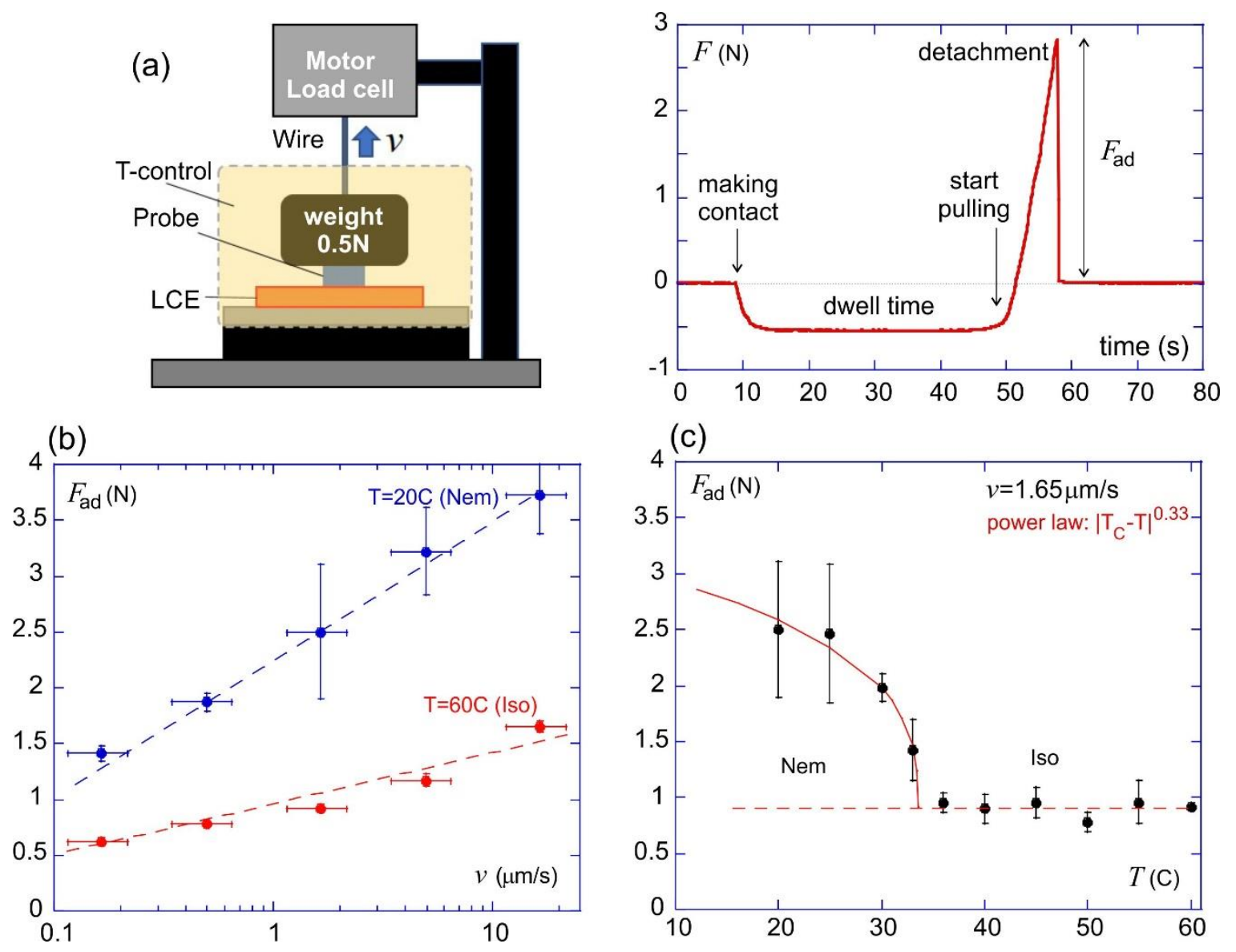

Figure 1. Dynamic adhesion on LCE: (a) Schematic of the probe-tack apparatus. After a flat glass surface with the squared shape (area of $25 \mathrm{~mm}^{2}$ ), probe, is first contacted with the LCE surface (thickness $d=0.1 \mathrm{~mm}$ ) at $0.5 \mathrm{~N}$ for $\sim 30 \mathrm{~s}$, the probe are pulled apart with a pulling speed of motor, $v_{\mathrm{m}}$, and force are monitored. A typical force vs. time curve at room at $v \sim 1.65$ $\mu \mathrm{m} / \mathrm{s}$ is shown on the right. Positive force indicates that required to detach the probe from the sample. Adhesive forces are read as the maximum of curves, labelled by $F_{\text {ad. }}$ (b) Plots of $F_{\text {ad }}$ vs. $v$, which is the probe speed estimated from the compliance of the apparatus, at RT and HT. (c) Plots of $F_{\text {ad }}$ vs. $T$ at $v \sim 1.65 \mu \mathrm{m} / \mathrm{s}$. The curve fitted to a power law toward the critical temperature, $\left(T_{\mathrm{c}}-T\right)^{1 / 3}$, is also shown for guidance. The arrow indicates the microscopic director direction. ( \pm s.d. are shown with error bars.) 


\section{WILEY-VCH}

The temperature-dependent adhesion has also been demonstrated on a different peeling experiment, called a $90^{\circ}$-peel test with constant force (Figure S3 and Movie in the Supporting Information). The adhesion is strong in the nematic phase; the peeling starts near $T_{\mathrm{NI}}$, and acceralates on further elevating $T$ in the isotropic phase, showing a good example of stimuliresponsive detachment on a smart soft adhesive. We have confirmed that this temperaturedependent adhesion is fully repeatable, at least after 10 heating-cooling cycles.

Before discussing the correlation between the observed adhesion response, and the basic material properties, such as the bulk viscoelasticity and the static surface tension, here we recall a known empirical expression ${ }^{[2,35]}$ for the work of adhesion $\Gamma$ per unit area of adhesive contact, which includes the viscoelastic dissipation effect;

$$
\Gamma\left(T, v_{\mathrm{c}}\right)=\Gamma_{0}(T)\left[1+f\left(T, v_{\mathrm{c}}\right)\right]
$$

Here $\Gamma_{0}$ is the threshold adhesion energy for zero crack propagation velocity (quasi-static peeling) between the adhesives and the contacting rigid probe, while $f\left(T, v_{c}\right)$ is the nondimensional factor related to the viscous energy loss during the interfacial crack propagation at speed $v_{c}$. Qualitatively, with the faster pulling speed, the higher force, and thus higher energy, should be required for the crack propagation to overcome the viscous resistance, which is incorporated into $f\left(T, v_{\mathrm{c}}\right)$.

Moreover, it has been proposed ${ }^{[36]}$ that the dissipative factor $f\left(T, v_{\mathrm{c}}\right)$ should be related to the bulk viscoelastic characteristics of the soft material via the loss factor $\tan \delta(T, \omega)$, where $\omega$ is the frequency of the applied oscillating strain in a dynamic-mechanical experiment. Although the actual $v_{c}$ should has a complicated form due to the stress concentration and nonlinear effects at the crack tip during detachment, ${ }^{[37]}$ assuming that $\omega$ is naively related to $v_{c}$, and to the probe speed $v$, as a first order approximation Equation (1) can be rewritten as,

$\Gamma(T, v) \sim \Gamma_{0}(T)[1+f(\tan \delta(T, \omega), c \omega)]$, 


\section{WILEY-VCH}

where function $f$ is a monotonic function of $\tan \delta$, which should depend on the geometry of peeling and modes of crack propagation, and $c$ is the characteristic length of displacement during crack propagation, connecting $v_{c}$ and $\omega$. This equation suggests that we can manipulate the temperature dependence of adhesion strength via $\Gamma_{0}(T)$ and/or via $\tan \delta(T, \omega)$.

Since the mechanical compliance of the apparatus in our adhesion tests was large, it was difficult to extract the pure adhesion energy of the sample represented by the integration $(d / A) \int_{0}^{\varepsilon_{\max }} F(\varepsilon) d \varepsilon$, where $\varepsilon$ is the strain applied to the sample, $\varepsilon_{\max }$ is that at detachment, both of them unknown. Thus, note that our adhesion experiments include the effect of the compliance of the apparatus. Since the adhesive force falls sharply after reaching $F_{\text {ad }}$ under the interfacial detachment regime, $\Gamma$ may crudely be related to $F_{\text {ad }}$ in order to estimate the work of adhesion per unit area: $\Gamma(T, v) \sim \frac{F_{\mathrm{ad}}(T, v)}{\sqrt{A}}$, where $\sqrt{A}$ is the characteristic size of the contact area. For example, at RT and $v_{\mathrm{m}}=0.1 \mathrm{~mm} / \mathrm{s}$ (Figure 1a) with $F_{\mathrm{ad}} \sim 2.5 \mathrm{~N}$ and $\sqrt{A} \sim 5 \mathrm{~mm}$, we estimate $\Gamma \sim 500 \mathrm{~N} / \mathrm{m}$, most of which should be the energy stored in the compliant apparatus, and then dissipated after detachment. Considering the typical ratio of the compliance of the sample to that of the apparatus, $r_{\mathrm{S} / \mathrm{A}} \sim 0.01$ (see Experimental), the pure adhesive energy density between the sample and the glass probe should be in the order of $\sim 5 \mathrm{~N} / \mathrm{m}$ in that case. Since this estimated value is much larger than the typical value provided by the Van der Waals interaction of the present elastomer-glass system, which is of the order $\sim 30 \mathrm{mN} / \mathrm{m}$, the viscoelastic effect $f(T, v)$ would be dominant in the work of adhesion: $f(\tan \delta(T, \omega), c \omega) \gg 1$. Then, we obtain the qualitative relationship,

$$
F_{\mathrm{ad}}(T, v) \propto \Gamma_{0}(T) f(\tan \delta(T, \omega), c \omega) .
$$

To discuss the main origin of the observed adhesion on our nematic LCE we separate the ratio into two factors: $R_{\mathrm{Nem} / \mathrm{Iso}}=\frac{F_{\mathrm{ad}}(\mathrm{RT}, v)}{F_{\mathrm{ad}}(\mathrm{HT}, v)}=R_{\mathrm{st}} R_{\mathrm{ve}}$, where the effects of static surface tension are 
captured by $R_{\mathrm{st}}=\frac{\Gamma_{0}(\mathrm{RT})}{\Gamma_{0}(\mathrm{HT})}$, while the viscoelastic dynamics is expressed by the ratio $R_{\mathrm{ve}}=$ $\frac{f(\tan \delta(\mathrm{RT}, \omega), c \omega)}{f(\tan \delta(\mathrm{HT}, \omega), c \omega)}$

Since $\Gamma_{0}(T)$ is related to the dispersion part of surface energies of the both two materials in contact, $\gamma_{\mathrm{LCE}}^{d}(T)$ and $\gamma_{\text {glass }}^{d}(T)$, the static work of adhesion between ideally flat interfaces, ${ }^{[38]}$ $w_{\mathrm{LCE}-\text { glass }}(T)$ is given by:

$\Gamma_{0}(T) \propto w_{\text {LCE-glass }}(T) \sim 2 \sqrt{\gamma_{\text {LCE }}^{d}(T) \gamma_{\text {glass }}^{d}(T)}$

As a simple expectation, the surface tension $\gamma^{d}(T)$ of typical polymeric materials ${ }^{[39]}$ is approximately $\sim 0.1 \mathrm{mN} / \mathrm{m} / \mathrm{K}$, the change in $\gamma^{d}(T)$ between RT and HT is small: $\sim 4 \mathrm{mN} / \mathrm{m}$. Very little temperature dependency of contact angles we measured on both LCE and the glass surface supports this estimation (Figure S4 in the Supporting Information). These contact angle measurements also confirm the typical values of the surface tension, at which the liquid can totally wet the surface of polymers and of glass as $\sim 30 \mathrm{mN} / \mathrm{m}$ and $\sim 20 \mathrm{mN} / \mathrm{m}$. Then, the ratio $R_{\mathrm{St}}=\frac{w_{\mathrm{LCE}-\text { glass }}(\mathrm{RT})}{w_{\mathrm{LCE}-\text { glass }}(\mathrm{HT})} \sim 1+\frac{4}{w_{\mathrm{LCE}-\text { glass }}(\mathrm{HT})}=1+\frac{4}{2 \sqrt{\gamma_{\mathrm{LCE}}^{d}(\mathrm{HT}) \gamma_{\text {glass }}^{d}(\mathrm{HT})}} \sim 1.09$. This is very much smaller than the observed ratio of adhesion strength in the two phases, so we again confirm that the static interfacial energies ${ }^{[8]}$ of the contact have a small effect on the present adhesion, i.e., viscoelastic dynamics, $R_{\mathrm{ve}}$, governs it. Nor can the surface roughness ${ }^{[40]}$ explain our observations: Even if the roughness exists, it should be much less than the scale of the nematic domain size of a few microns. ${ }^{[15]}$ Moreover, the low storage modulus of an elastomer, less than MPa in both phases as shown in Figure 2a, would result in an almost ideal contact with a hard and flat glass surface. 

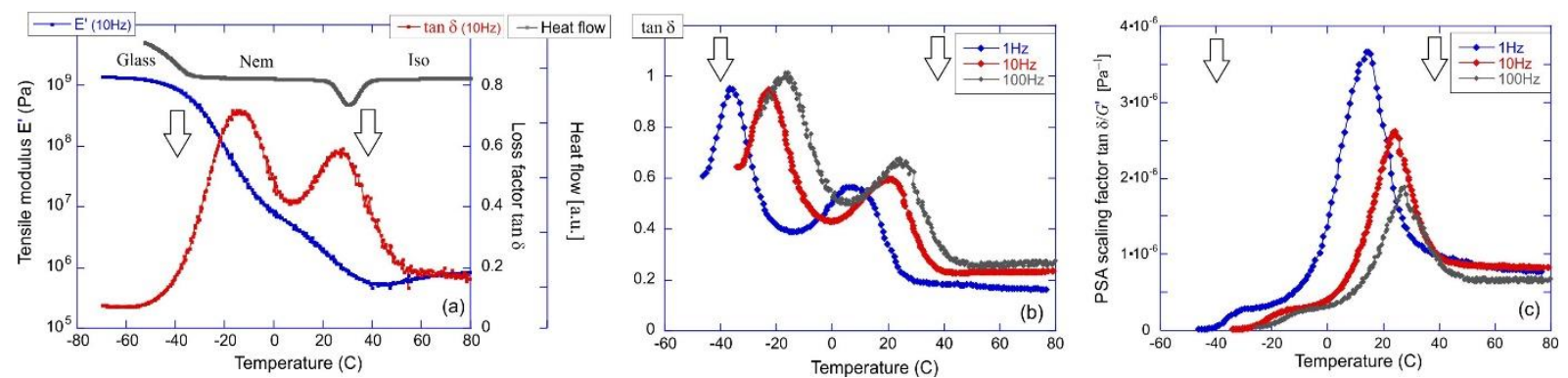

Figure 2. Dynamic soft elasticity in LCE: (a) The tensile storage modulus $E^{\prime}$, and the loss factor $\tan \delta$, at fixed frequency $\omega=10 \mathrm{~Hz}$, on sample cooling at $3^{\circ} \mathrm{C} / \mathrm{min}$. The graph also shows the DSC scan, on cooling at $5^{\circ} \mathrm{C} / \mathrm{min}$, illustrating the nematic and the glass transition temperatures (marked by arrows in all three plots). (b) The frequency dependence of the dynamic transitions, illustrated by the loss factor $\tan \delta$, on sample cooling at $3^{\circ} \mathrm{C} / \mathrm{min}$. (c) The frequency-dependent scaling factor $\tan \delta / G^{\prime}$ for PSA, illustrating the major role of the internal dissipation in the nematic phase.

The dynamic-mechanical test results shown in Figure 2 indicate the characteristic large increase of the loss factor $\tan \delta$ in the nematic phase (the effect of dynamic soft elasticity). Since we have assumed a very simple approximation for the relation between the frequency $\omega$ and $v$ in Equation (2), it is possible to evaluate only the tendency of how $f\left(T, v_{\mathrm{c}}\right)=$ $f(\tan \delta(T, \omega), c \omega)$ changes with increasing $v$. By increasing $\omega$, which roughly corresponds to the increase of $v, \tan \delta$ within the nematic range also increases (Figure 2b). This directly correlates with the increase of adhesion on increasing $v$ (Figure 1b). Although there still a need for detailed experiments and a proper theory for the full understanding of this problem, we may conclude that the observed strong temperature dependence of adhesion is attributed to the drastic changes in the viscoelastic loss in the LCE.

The DMA tests also show large values of $\tan \delta$ at low temperatures, which corresponds to the glass transition region (Figure 2). Although it is tempting to follow the previous logic, and suggest that the glass transition region is also useful to increase adhesion, ${ }^{[29]}$ in practice, 


\section{WILEY-VCH}

this is not the case. There is a lot of literature in the general field of pressure sensitive adhesives ${ }^{[1,2]}$ where the scaling gauge is developed to estimate the performance of materials. It is established that the ratio $\frac{\tan \delta(T, \omega)}{G^{\prime}(T, \omega)}$ is most useful for this purpose ${ }^{[41,42]}$ (see Figure 2c). Therefore, the large $G^{\prime}$ in the glassy region suppresses the effective adhesion strength, in spite of the $\tan \delta$ rise at the low temperature. In contrast, the storage modulus changes little between the nematic and isotropic phases of the elastomer. If anything, there is a decrease of $G^{\prime}$ and $E^{\prime}$ in the nematic phase close to the $T_{\mathrm{NI}}$ (as shown in Figure 2a), which further enhances the effect on adhesion. This discussion suggests that the LCE in the nematic phase is a good candidate as a pressure sensitive adhesive and, additionally, the high adhesion can be switched-off on changing the phase.

In conclusion, we report that the adhesion of a nematic LCE can be changed drastically by small changes in temperature, when it crosses between the nematic and isotropic phases, exploiting the inherent bulk viscoelastic properties of the nematic LCEs. Needless to say, the present material, showing the relatively low adhesion strength suitable for PSAs, requires optimization of its properties (Table 1) toward any practical applications, which is beyond the scope of this work. It is possible to modulate $T_{\mathrm{NI}}, E^{\prime}, E^{\prime \prime}$, and the surface chemistry through the variation of material components, and a large literature exists on this subject. ${ }^{[10]}$ For instance, $T_{\mathrm{NI}}$ can be increased by decreasing the amount of non-mesogenic spacers, and $E^{\prime}$ can be lowered by decreasing the amount of the crosslinker. The nematic-isotropic phase transition can also be induced by other stimuli, for example light and chemicals, ${ }^{[10]}$ by incorporating the molecular units sensitive to them in the polymer formulation. This makes the reported mechanism of reversible viscoelastic damping be applicable in a broad class of smart switchable soft adhesives toward, household, biomedical, and manufacturing applications. 


\section{WILEY-VCH}

\section{Experimental Section}

Materials and preparation of LCE: For preparation of LCE, we followed the methods reported previously, ${ }^{[32-34]}$ in which two-step crosslinking reactions, a thiol-acrylate Michael addition and a photoinduced radical polymerization of diacrylates, with slight modifications. The diacrylate monomer, 1,4-bis-[4-(6-acryloyloxyhexyloxy)benzoyloxy]-2-methylbenzene (RM82), was purchased from Wilshire Technologies (Figure S1). The diacrylate spacer, tri(propylene glycol), and two thiol monomers: 2,2'-(ethylenedioxy) diethanethiol (EDDET) and pentaerythritol tetrakis (3-mercaptopropionate) (PETMP), were purchased from Sigma Aldrich. Triethylamine (TEA, Sigma Aldrich) was used as the Michael-addition catalyst. As the photoinitiator, the Irgacure2959 from BASF was used. As the radiacal scavenger, butylated hydroxytoluene (BHT, from Sigma Aldrich) was used to supress the unwanted radical polymerization of acrylates. All chemicals were used in their as-received condition with no purification. At the specific molar functional ratio shown in Figure S1, RM82, TPGDA, EDDET and PETMP were weighed, Irgacure2959 (0.2wt\%), and BHT (0.5wt\%) were added. After the mixture was gently mixed at an elevated $T \sim 70^{\circ} \mathrm{C}$ for $\sim 10 \mathrm{~min}$, TEA $(1.5 \mathrm{wt} \%)$ was added to start the Michel-addition reaction between thiol and acrylate groups. The mixture was molded between two glass slides with spacers with thickness of $0.1 \mathrm{~mm}$ or $1 \mathrm{~mm}$ at $70^{\circ} \mathrm{C}$ (isotoropic phase) for overnight. The sample was then cooled down to RT and UV light (365 $\mathrm{nm}$ ) was irradiated for $20 \mathrm{~min}$ to finalize the crosslinking reaction between the rest of the acrylates in order to ensure the genesis of the Nem phase at RT for the final product. For WAXS measurment, a sample with UV light irradiated under uniaxial tensile strain (0.5) was also prepared as a reference. The samples were annealed at $80^{\circ} \mathrm{C}$ in vacuum oven for 12 hours for evaporation of TEA before further characterizations.

Adhesion tests: The probe-tack test ${ }^{[2]}$ used here consists of making contact between the flat surface of a solid probe and the thin adhesive sample layer coated on a rigid glass substrate, and 


\section{WILEY-VCH}

measuring the force $F_{\text {ad }}$ required to detach the probe at a constant pulling speed by motor $v_{\mathrm{m}}$. The LCE sample with the thickness $d=0.1 \mathrm{~mm}$ supported by a glass slide was prepared by careful peeling of one of the two slide glassed used during the crosslinking reactions of the LCE described in the sample preparation method. Adhesion tests were carried out under controlled $T$ with a home-made probe-tack set-up. The load cell $(150 \mathrm{~N})$ for the measurement of the normal force $F$ is composed in series with the probe with weight, which applies pre-load $(0.5$ $\mathrm{N}$ for $30 \mathrm{~s}$ dwell time) before detachment action. Since the dwell time is known to affect the adhesion strength, especially on the viscous/plastic materials, we have chosen the dwell time to avoid the time scales in which the fast relaxation occurs (See Figure S5); the $85 \%$ of transient stress has already fully relaxed at $30 \mathrm{~s}$ in the present LCE at nematic phase. A flat glass surface with the square area of $25 \mathrm{~mm}^{2}$ was used as the probe. After each probe-tack test, the probe surface was cleaned with ethanol. Each test was repeated at least four times $(N=4)$ and the average of the maximum force reads for detachment was determined as $F_{\text {ad }}$ for each condition. The compliance $K_{\mathrm{A}} \sim 0.2 \mathrm{~mm} / \mathrm{N}$ of the apparatus around $F \sim 2 \mathrm{~N}$ was estimated by a blank test (without sample and with the probe fixed to the substrate). This value was used to estimate the actual probe speed $v$ relative to the sample as follows. Results of the dynamic mechanical tests showed that the storage modulus, $E^{\prime}$ ( $\sim$ Young's modulus, $E$ ), of the sample was approximately $E \sim 1.2 \pm 0.5 \mathrm{MPa}$ within the range between RT and HT. With the contact area $A=25 \mathrm{~mm}^{2}$ and sample thickness $d=0.1 \mathrm{~mm}$, the effective compliance of the sample before detachment $K_{\mathrm{S}}=$

$\frac{d}{E A} \sim 0.0033 \mathrm{~mm} / \mathrm{N}(0.0023 \sim 0.0057 \mathrm{~mm} / \mathrm{N}$ considering the error in $E)$. Since $K_{\mathrm{A}} \gg K_{\mathrm{S}}, v$ is much smaller than the applied pulling speed of the motor, $v_{\mathrm{m}}$, and is estimated as $v=r_{\mathrm{S} / \mathrm{A}} v_{\mathrm{m}}=$ $\left(K_{\mathrm{S}} / K_{\mathrm{A}}\right) v_{\mathrm{m}} \sim 0.0165 v_{\mathrm{m}}\left(0.0115 v_{\mathrm{m}} \sim 0.0285 v_{\mathrm{m}}\right.$ considering the error in $\left.E\right)$. Although the absolute values of $v$ are not accurate, they are reliable regarding the order magnitude and the relative differences, and thus, are useful for comparison to other general adhesion results. 


\section{WILEY-VCH}

Dynamic scanning callorimetry (DSC): For differential scanning calorimetry (DSC, DSC4000

PerkinElmer), samples with approximately $10 \mathrm{mg}$ were loaded into standard aluminium DSC pans. The samples were heated to $90^{\circ} \mathrm{C}$ at $10^{\circ} \mathrm{C} / \mathrm{min}$, held isothermally for $5 \mathrm{~min}$, and cooled to $-60^{\circ} \mathrm{C}$ at $5^{\circ} \mathrm{C} / \mathrm{min}$ to acquire the data. $T_{\mathrm{NI}}$ can be found at local minimum of the endothermic peak. The sample was run three times $(N=3)$.

Dynamic mechanical analysis (DMA): The dynamic mechanical tests were performed on a Viscoanalyser-4000, Metravib, in the shear sandwich and the tension modes, with samples of 1 mm film thickness. For the shear mode, a circular sample (diameter of $10 \mathrm{~mm}$ ) was used. The simple shear strain of $2 \%$ was applied at frequencies of 1,10 , and $100 \mathrm{~Hz}$. For tension mode, a rectangular sample (effective length of $15 \mathrm{~mm}$ and width of $5 \mathrm{~mm}$ ) was used. The simple strain of $0.2 \%$ was applied at frequency of $10 \mathrm{~Hz}$. Data were acquired on cooling at the rate of $3^{\circ} \mathrm{C} / \mathrm{min}$ from 80 to typically $-40^{\circ} \mathrm{C}$ (and to $-60^{\circ} \mathrm{C}$ in the tension mode).

Wide angle $X$-ray scattering (WAXS): The phase of the present LCE at RT was characterized using a Philips diffractometer using a Philips Copper target (PW-2233/20) with the wavelength of $0.154 \mathrm{~nm}$. The distance between the sample and the imaging area was $100 \mathrm{~mm}$.

Contact angle measurements: Contact angles of liquids were measured on a LCE sample and the slide glass used as the probe for adhesion tests. Propylene glycol, ethylene glycol and glycerol were purchased from Sigma-Aldrich. Each $10 \mathrm{~mL}$ of liquid was gently placed on the sample surface under $T$-control and the image was taken $20 \sim 40 \mathrm{~s}$ after the deposition. The angles of each four droplets $(N=4)$ were analyzed using a software (ImageJ) and their averaged values were used for discussion.

\section{Supporting Information}

Supporting Information is available from the Wiley Online Library or from the author. 


\section{WILEY-VCH}

\section{Acknowledgements}

This work was supported by the European Research Council grant No: 786659 and partly by JSPS KAKENHI Grant Number JP17K18862. The authors are grateful for many useful communications and advice from Yasuo Norikane, Yufeng Yue, Haruhisa Akiyama and Alexandra Gablier.

Received: ((will be filled in by the editorial staff))

Revised: ((will be filled in by the editorial staff)) Published online: ((will be filled in by the editorial staff))

\section{References}

[1] I. Benedek, M. M. Feldstein, Handbook of Pressure-Sensitive Adhesives and Products: Fundamentals of Pressure Sensitivity, CRC Press, 2008.

[2] C. Creton, M. Ciccotti, Rep. Prog. Phys. 2016, 79, 46601.

[3] M. Kamperman, A. Synytska, J. Mater. Chem. 2012, 22, 19390.

[4] S. Reddy, E. Arzt, A. Del Campo, Adv. Mater. 2007, 19, 3833.

[5] P. C. Lin, S. Vajpayee, A. Jagota, C. Y. Hui, S. Yang, Soft Matter 2008, 4, 1830.

[6] E. P. Chan, E. J. Smith, R. C. Hayward, A. J. Crosby, Adv. Mater. 2008, 20, 711.

[7] T. Ohzono, K. Teraoka, Soft Matter 2017, 13, 9082.

[8] G. De Crevoisier, P. Fabre, J. M. Corpart, L. Leibler, Science 1999, 285, 1246.

[9] K. Cho, J. H. Cho, S. Yoon, C. E. Park, J. C. Lee, S. H. Han, K. B. Lee, J. Koo, Macromolecules 2003, 36, 2009.

[10] Y. Gao, K. Wu, Z. Suo, Adv. Mater. 2019, 31, 1.

[11] H. Akiyama, M. Yoshida, Adv. Mater. 2012, 24, 2353.

[12] S. Ito, H. Akiyama, R. Sekizawa, M. Mori, M. Yoshida, H. Kihara, ACS Appl. Mater. Interfaces 2018, 10, 32649.

[13] E. M. Warner, M., Terentjev, Liquid Crystal Elastomers, Oxford Univ. Press, 2007.

[14] L. Petridis, E. M. Terentjev, Phys. Rev. E 2006, 74, 1.

[15] S. V. Fridrikh, E. M. Terentjev, Phys. Rev. E 1999, 60, 1847.

[16] J. Küpfer, H. Finkelmann, Mocromolecular Rapid Commun. 1991, 12, 717.

[17] A. R. Tajbakhsh, E. M. Terentjev, Eur. Phys. J. E 2001, 6, 181. 


\section{WILEY-VCH}

[18] T. J. White, D. J. Broer, Nat. Mater. 2015, 14, 1087.

[19] Y. Yu, M. Nakano, T. Ikeda, Nature 2003, 425, 145.

[20] T. Guin, M. J. Settle, B. A. Kowalski, A. D. Auguste, R. V Beblo, G. W. Reich, T. J. White, Nat. Commun. 2018, 1.

[21] Y. Yue, Y. Norikane, R. Azumi, E. Koyama, Nat. Commun. 2018, 9, 3234.

[22] G. Babakhanova, T. Turiv, Y. Guo, M. Hendrikx, Q. H. Wei, A. P. H. J. Schenning, D. J. Broer, O. D. Lavrentovich, Nat. Commun. 2018, 9, 1.

[23] S. M. Clarke, A. R. Tajbakhsh, E. M. Terentjev, C. Remillat, G. R. Tomlinson, J. R. House, J. Appl. Phys. 2001, 89, 6530.

[24] E. M. Terentjev, A. Hotta, S. M. Clarke, M. Warner, G. Marrucci, J. M. Seddon, H. S. Sellers, Philos. Trans. R. Soc. A Math. Phys. Eng. Sci. 2003, 361, 653.

[25] S. M. Clarke, A. R. Tajbakhsh, E. M. Terentjev, M. Warner, Phys. Rev. Lett. 2001, 86, 4044.

[26] D. R. Merkel, R. K. Shaha, C. M. Yakacki, C. P. Frick, Polymer 2019, 166, 148.

[27] E. M. Terentjev, M. Warner, Eur. Phys. J. E 2001, 4, 343.

[28] O. Stenull, T. C. Lubensky, Phys. Rev. E 2004, 69, 13.

[29] N. Lakhera, A. Graucob, A. S. Schneider, E. Kroner, E. Arzt, C. M. Yakacki, C. P. Frick, Int. J. Adhes. Adhes. 2013, 44, 184.

[30] D. H. Kaelble, J. Adhes. 1969, 1, 102.

[31] D. R. Corbett, J. M. Adams, Soft Matter 2013, 9, 1151.

[32] D. P. Nair, N. B. Cramer, J. C. Gaipa, M. K. McBride, E. M. Matherly, R. R. McLeod, R. Shandas, C. N. Bowman, Adv. Funct. Mater. 2012, 22, 1502.

[33] C. M. Yakacki, M. Saed, D. P. Nair, T. Gong, S. M. Reed, C. N. Bowman, RSC Adv. 2015, 5, 18997.

[34] M. O. Saed, R. H. Volpe, N. A. Traugutt, R. Visvanathan, N. A. Clark, C. M. Yakacki, Soft Matter 2017, 13, 7537. 


\section{WILEY-VCH}

[35] A. N. Gent, J. Schultz, J. Adhes. 1972, 3, 281.

[36] D. Maugis, J. Phys. D. Appl. Phys. 2001, 11, 1989.

[37] B. Lorenz, B. A. Krick, N. Mulakaluri, M. Smolyakova, S. Dieluweit, W. G. Sawyer, B. N. J. Persson, J. Phys. Condens. Matter 2013, 25, 225004.

[38] J N Israelachvili, Intermolecular and Surface Forces, Academic Press, 2011.

[39] D. Y. Kwok, L. K. Cheung, C. B. Park, A. W. Neumann, Polym. Eng. Sci. 1998, 38, 757.

[40] D. Liu, L. Liu, P. R. Onck, D. J. Broer, Proc. Natl. Acad. Sci. 2015, 112, 3880.

[41] T. Wang, C. H. Lei, A. B. Dalton, C. Creton, Y. Lin, K. A. S. Fernando, Y. P. Sun, M. Manea, J. M. Asua, J. L. Keddie, Adv. Mater. 2006, 18, 2730.

[42] T. Wang, P. J. Colver, S. A. F. Bon, J. L. Keddie, Soft Matter 2009, 5, 3842. 